\title{
Genetic Characterization of Porcine Circovirus Type 2 from Pigs with Porcine Circovirus Associated Diseases in Argentina
}

\author{
Ariel Pereda, ${ }^{1}$ Pablo Piñeyro, ${ }^{2}$ Ana Bratanich, ${ }^{3}$ María Alejandra Quiroga, ${ }^{2}$ \\ Danilo Bucafusco, ${ }^{3}$ María Isabel Craig, ${ }^{1}$ Javier Cappuccio, ${ }^{2}$ Mariana Machuca, ${ }^{2}$ \\ Agustina Rimondi, ${ }^{1}$ Marina Dibárbora, ${ }^{1}$ Hector Ramón Sanguinetti, ${ }^{4}$ \\ and Carlos Juan Perfumo ${ }^{2}$ \\ ${ }^{1}$ Laboratorio Aves y Porcinos, Instituto de Virologia CICVyA, Instituto Nacional de Tecnologia Agropecuaria (INTA), \\ CC25, 1712 Castelar, Buenos Aires, Argentina \\ ${ }^{2}$ Cátedra de Patología Especial, Departamento de Ciencias Pre-clínicas, Facultad de Ciencias, Veterinarias, \\ Universidad Nacional de La Plata, Casilla de Correo N²96, Calle 60 y 118 S/N, 1900 La Plata, Buenos Aires, Argentina \\ ${ }^{3}$ Area de Virología, Facultad de Ciencias, Veterinarias, Universidad de Buenos Aires, Chorroarín 280, C1427CWO CABA, Argentina \\ ${ }^{4}$ Servicio Nacional de Sanidad y Calidad Agroalimentaria, Dirección de Laboratorio Animal, Avenida Fleming 1653, \\ 1640 Martínez, Buenos Aires, Argentina
}

Correspondence should be addressed to Carlos Juan Perfumo, cjperfumo@fcv.unlp.edu.ar

Received 4 March 2011; Accepted 5 April 2011

Academic Editors: M. Benko, R. Thanawongnuwech, and S. Vilcek

Copyright ( 2011 Ariel Pereda et al. This is an open access article distributed under the Creative Commons Attribution License, which permits unrestricted use, distribution, and reproduction in any medium, provided the original work is properly cited.

Porcine circovirus type 2 (PCV-2) has been associated with syndromes grouped by the term porcine circovirus associated diseases (PCVAD). The PCV-2 isolates have been grouped into two major groups or genotypes according to their nucleotide sequence of whole genomes and/or ORF-2: PCV-2b, which have, in turn, been subdivided into three clusters (1A-1C), and PCV-2a, which has been subdivided into five clusters (2A-2E). In the present study, we obtained 16 sequences of PCV-2 from different farms from 2003 to 2008, from animals with confirmatory diagnosis of PCVAD. Since results showed an identity of $99.8 \%$ among them, they were grouped within a common cluster $1 \mathrm{~A}-\mathrm{B}$. This preliminary study suggests a stable circulation of PCV-2b among the Argentinean pig population.

\section{Introduction}

Porcine circovirus (PCV) is a small nonenveloped virus that contains a single-stranded circular DNA of about $1.76 \mathrm{~kb}$. PCV was originally isolated as a noncytopathic contaminant of the PK-15 cell line [1] and was classified as a member of Circoviridae family, genus Circovirus, based on its morphological and genomic characteristics $[2,3]$.

Two phenotypically different but genetically related strains of PCV have been identified in swine. PCV-1 was first detected as a contaminant of the porcine kidney PK-15 cell line [4] whereas PCV-2 has been associated with postweaning multisystemic wasting syndrome (PMWS) $[5,6]$, porcine dermatitis and nephropathy syndrome (PDNS) [7], proliferative necrotizing pneumonia [8], and reproductive disorders [9], all of them included by the term porcine circovirus associated diseases (PCVAD) [10]. PMWS is an emerging disease in pigs first described in a swine herd in Canada in 1991 [5] and also endemic in many swine-producing countries. In Argentina, PMWS was first reported in 2002 [11].

Diagnosis of PMWS is based on the presence of compatible clinical signs [12], characteristic histopathological lesions, and detection of PCV-2 antigen within typical lesions $[10,13]$.

The genomic structure of PCV-2 consists of two intergenic regions flanked by three open reading frames (ORFs): ORF-1, which encodes two replication proteins (Rep and Rep'), ORF2, which encodes the Cap protein containing the immunoreactive epitopes and is more variable at nucleotide sequence than ORF1, and ORF3, which encodes a proapoptotic protein [14]. Currently, PCV-2 genotype definition and 
nomenclature has been proposed according to the genome sequence of the whole genome and/or ORF2 [15, 16]. Five genotypes have been identified to date $[17,18]$ : PCV-2a and PCV-2b, which correspond to the main phylogenetic groups, PCV-2c, which has been described only in Denmark, and PCV-2d and 2e, which have been described in China [18]. PCV-2a has been further subdivided into five clusters (2A2E) [15] and PCV-2b into three clusters (1A-1C). Since 2005, high mortality outbreaks of PMWS reported in North America have been associated with PCV-2b $[19,20]$. In South America, only Brazil has carried out genotyping studies on PCV-2. Phylogenetic studies have shown that PCV-2 isolates belong to PCV-2b and PCV-2a $[21,22]$. The objectives of this study were to compare the nucleotide and amino acid sequences of the PCV-2 ORF2 identified in lymph nodes from pigs with PCVAD from different herds of Argentina between 2003 and 2008 .

\section{Materials and Methods}

2.1. Immunolabelling for $P C V-2$. Immunohistochemistry was performed with a polyclonal anti-PCV-2 antibody (VMRD, Inc., WA, USA, 210-70 PCRV). Briefly, tissue sections were deparaffinized with xylene and rehydrated through graded alcohols. Slides were flooded for $15 \mathrm{~min}$ with $3 \% \mathrm{H}_{2} \mathrm{O}_{2}$ to remove endogenous peroxidase activity. Tissues were rinsed for $5 \mathrm{~min}$ in $0.1 \mathrm{M}$ PBS $(\mathrm{pH} 7.5)$ and then incubated with preheated $0.05 \%$ protease XIV for 40 minutes. Tissue sections were rinsed in PBS and flooded with $0.5 \%$ skim milk in PBS for $20 \mathrm{~min}$ at room temperature. PCV-2 antibody was used at a $1 / 200$ dilution in $0.1 \mathrm{M}$ PBS, and incubated for 1 hour at $37^{\circ} \mathrm{C}$. Biotinylated $\mathrm{G}$ protein $(1 / 500)$ was used as a secondary antibody and was incubated for $40 \mathrm{~min}$ at room temperature. Streptavidinperoxidase (LSAB2 System HRP K0673, DAKO Laboratories Co., CA, USA) was applied for $15 \mathrm{~min}$ at room temperature. Sections were finally incubated in diaminobenzidinehydrogen peroxide solution for $8 \mathrm{~min}$ and counterstained with Harris' haematoxylin. Positive and negative controls were used. Immunohistochemistry were graded based on the intensity of immunolabelling as follows: $+=$ slight, $++=$ moderate, and $+++=$ abundant.

2.2. Samples and DNA Extraction. The samples used in this study corresponded to pig submissions to the Diagnostic Pathology Service, Faculty of Veterinary Science, La Plata National University, Argentina, from 2003 to 2005, and to the Virology laboratory from the Faculty of Veterinary Science of Buenos Aires from 2007 to 2008. These samples originated from 101 lymph nodes from pigs (36 to 140 days old) with confirmatory PCVAD diagnosis by immunohistochemistry (IHC). Samples from 2003 to 2005 were pooled in nine groups (6-10 samples/pool) according to the farm, province, and year of collection. Samples from 2007 to 2008 were processed individually (Table 1). Samples were homogenized with a mortar and pestle, in some cases after deparaffinization with xylene, and kept at $-80^{\circ} \mathrm{C}$ until processed.
DNA extraction was carried out using the QIAamp DNA mini kit (Qiagen, Valencia, USA) in accordance with the manufacturer's instructions.

2.3. Identification of $P C V-2$ and Amplification of the ORF2. PCV-2 specific DNA was amplified by PCR with GoTaq (Promega) in a MyCycler thermocycler (BioRad, UK). The ORF2 from positive samples was further amplified from nucleotides 998 to 1757 (PCV-2b genome; GenBank Accession Number, AF112862), using the protocol reported by Fort et al. [23]. In this protocol, two internal primers (capARv 5'-ACCCTTTGAATACTACAGA-3' and capBFw $5^{\prime}$-GGGAGGAGTAGTTTACATA- $3^{\prime}$ ) were added to make the sequencing process more reliable, since it amplifies the whole ORF2 in two partially overlapping fragments. Cycling conditions were as follows: $94^{\circ} \mathrm{C} 5 \mathrm{~min}, 35$ cycles of $45 \mathrm{sec}$ at $94^{\circ} \mathrm{C}, 45 \mathrm{sec}$ at $42^{\circ} \mathrm{C}$ and $1 \mathrm{~min}$ at $72^{\circ} \mathrm{C}$, and a final extension cycle of $7 \mathrm{~min}$ at $72^{\circ} \mathrm{C}$.

2.4. Nucleotide Sequencing and Phylogenetic Analyses. ORF2 amplified fragments were sequenced with the BigDye terminator kit (Applied Biosystems, Foster City, CA, USA) on an ABI 3500xL Genetic Analyzer (Applied Biosystems, Foster City, CA, USA). Sequence information was derived from overlapping sequences covered by forward and reverse primers. Sequences are available at GenBank, accession numbers EU980087 through EU980094. Then, all available isolates obtained were included in a multiple alignment using CLUSTAL X Version 1.8.3 program [24], and the percent identities were calculated. The phylogenetic trees, calculated by the neighbor-joining method, were computed with the DNADIST and NEIGHBOR modules of the PHYLIP package [25]. Bootstrapping values (1000 replicates) were calculated with the SEQBOOT, DNADIST, NEIGHBOR, and CONSENSE modules. Branches with bootstrapping values $\geq 70$ were considered significant, corresponding to a confidence interval $\geq 95 \%$ [26]. For visualization and printing of the trees, the TREEVIEW program, Version 1.6.6 was used [27]. To root the phylogenetic tree, we used the sequence of a porcine circovirus 1 (GenBank accession number AY184287).

\section{Results}

3.1. Detection of PCV-2 by PCR. The signs that predominated in the affected pigs were principally wasting in which the animals presented slow growth, lethargy, anorexia, cachexia, diarrhea, and dyspnea (Table 1). From those animals, 101 lymph nodes were processed and 58 (57.4\%) were positive for PCV-2 by IHC. Samples from 2003 to 2005 were grouped in 9 pools and $8(88.8 \%)$ were positive for PCV-2 by PCR. This correlates with the lack of IHC positive samples in the negative pool. Seven samples from 2007 and 2008 were processed individually and all resulted positive by PCR.

3.2. Nucleotide and Amino Acid Sequence of ORF2. The entire Cap coding region of 16 Argentinean samples was amplified 
TABLE 1: PCV-2-positive samples and sample pools included in this study.

\begin{tabular}{|c|c|c|c|c|}
\hline Identification & Province location & Main clinical signs associated with PCVAD & Year & Accession number \\
\hline $1^{*}$ & Cordoba & Wasting & 2003 & EU980089 \\
\hline $3^{*}$ & Buenos Aires & Cachexia & 2004 & EU980094 \\
\hline $5^{*}$ & Buenos Aires & Cachexia, dyspnoea & 2005 & EU980087 \\
\hline $6^{*}$ & Buenos Aires & Diarrhea, dyspnea, pallor & 2004 & EU980088 \\
\hline $8^{*}$ & Buenos Aires & Dyspnoea & 2003 & EU980090 \\
\hline $10^{*}$ & Buenos Aires & Wasting & 2003 & EU980092 \\
\hline $11^{*}$ & Buenos Aires & Wasting, diarrhea, nervous signs & 2003 & EU980093 \\
\hline $13^{*}$ & Santa Fé & Dyspnoea, fever & 2005 & EU980091 \\
\hline Salto & Buenos Aires & Wasting & 2005 & EF458306 \\
\hline 6743 & Santa Fé & Wasting & 2007 & HM565918 \\
\hline 6893 & Buenos Aires & Wasting & 2007 & HM565920 \\
\hline 7013 & Santa Fé & Wasting & 2008 & HM565921 \\
\hline 7040 & Buenos Aires & Wasting & 2007 & HM565922 \\
\hline 7108 & Buenos Aires & Wasting & 2007 & HM565923 \\
\hline 7109 & Santa Fé & Wasting & 2008 & HM565924 \\
\hline 7151 & Buenos Aires & Wasting & 2008 & HM565925 \\
\hline
\end{tabular}

${ }^{*}$ Corresponds to sample pools.

and sequenced (Table 1). The sequence analysis showed that they contained $699 \mathrm{nt}$, and no gaps were detected. Comparison of the sequences analyzed revealed an identity of $99.8 \%$ among them. All sequences presented the typical PCV-2 1486 motif TcAaacCCC/CGC. At the amino acid level, only two polymorphic sites were detected in the following samples: in 7109 we found K63R and in 7013, 7108, and 7198 we found T190A. Only the K63R mutation was localized in an immunogenic domain, specifically at the domain A.

These sequences were aligned with cap gene sequences from PCV-2 strains available in the GenBank database from different countries. All Argentinean amino acid sequences showed high identity with the sequences grouped in genotype $2 \mathrm{~b}$.

3.3. Phylogenetic Analysis. The phylogenetic tree obtained with the Argentinean and reference PCV-2 strain sequences showed two main clusters wellsupported by bootstrap analysis (Figure 1). All the Argentinean sequences analyzed grouped together in one cluster and were closely related to sequences from genotype PCV-2b isolates, and more specifically to cluster $1 \mathrm{~A}-\mathrm{B}$. In this last case, the low bootstrap values made the differentiation of the two clusters uncertain. We propose that they should be joined in a common cluster 1AB. This cluster grouped also isolates from Europe (Hungary, France, Austria, and the Netherlands), Asia (China), and North America (USA and Canada). In the other cluster, named 1C, only grouped sequences from Asia (China).

\section{Discussion}

Recent phylogenetic analysis of complete PCV-2 genomic sequences obtained from GenBank [15] and full-length
ORF2 sequences [16] resulted in two major genogroups: 2a and $2 b$, which are circulating worldwide, $2 c$, which have been sporadically reported in the 80 s [17], and $2 \mathrm{~d}$ and $2 \mathrm{e}$, recently described in China [18]. The significance of these differences in terms of disease and/or geographic location is currently unknown. However, the severe re-emergence of PCVAD in North America in 2005 coincided with the identification of the PCV-2b genotype [19]. In addition, an epizootic of PMWS in Switzerland was found to be associated with PCV2b while PCV-2a was identified in single cases of PMWS prior to 2003 [28]. Furthermore, experimental studies in gnotobiotic pigs with or without immunostimulation using PCV-2b alone have shown only moderate lesions in various organs, thus indicating that PCV-2b-stimulated pigs became infected but did not progress to a disease state [29]. However, another study using gnotobiotic pigs and cell culture-derived PCV-2a and PCV-2b clones showed that both were able to induce severe disease in this model [30]. A study has highlighted the importance of the order of infection in which gnotobiotic pigs inoculated with PCV-2b seven days after a PCV-2a infection, but not vice versa, cause PMWS [29]. However, when SPF instead of gnotobiotic pigs was used, results were negative [31]. Unfortunately, sequence studies with PCV-2a followed with PCV-2b in conventional pigs are still lacking.

The above results are indicative of sometimes contradicting information regarding PCV-2 genotyping and its relationship with disease condition. Although it is generally believed that the causes of illness depend on unknown factors, more information related to PCV-2 genotyping could help for a better understanding of the pathogenic mechanisms of PCV-2.

In the present study, PCV-2 sequences obtained from 16 different farms belonging to the main pig-producing provinces of Argentina were analyzed to determine the 


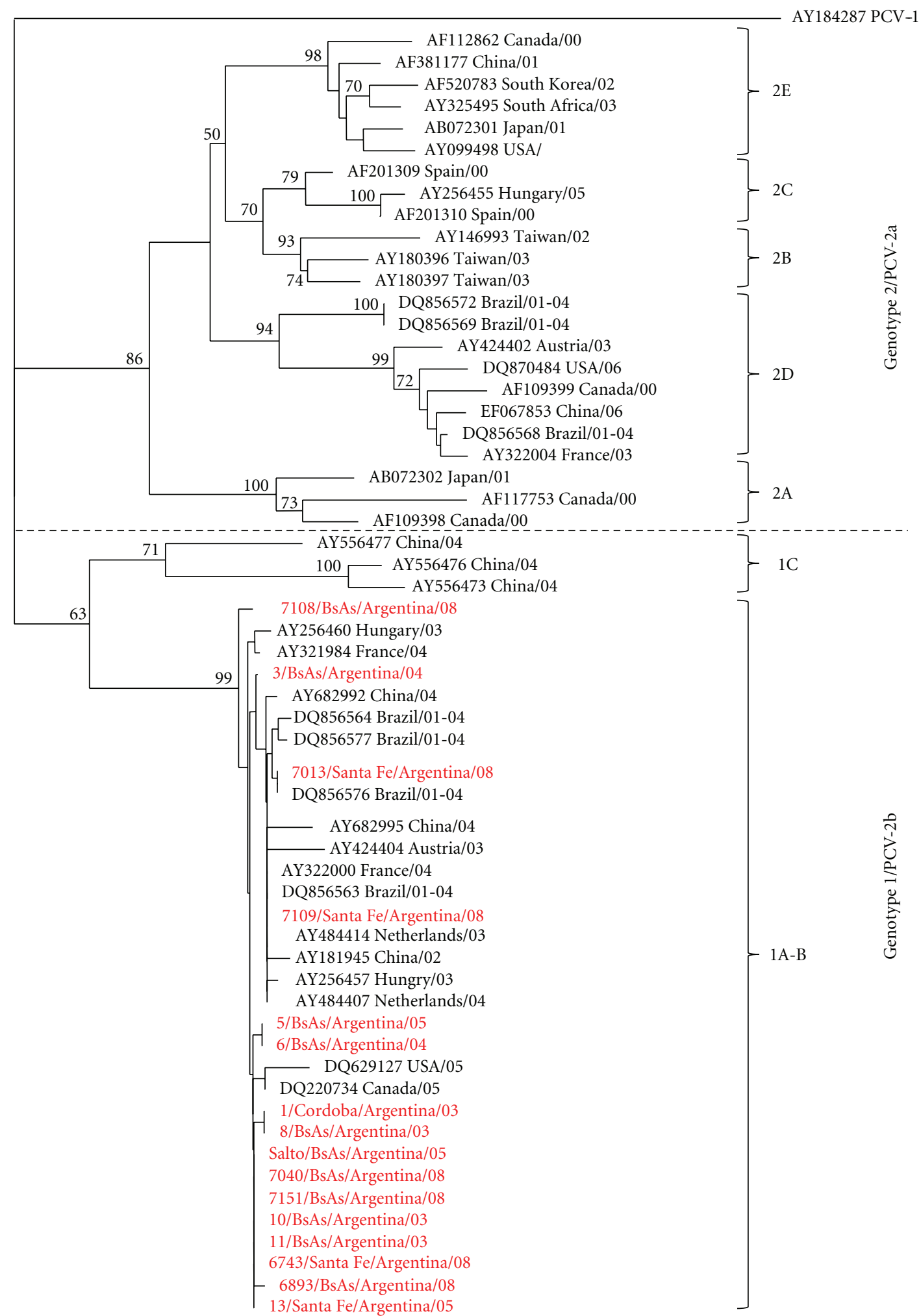

0.1

FIGURE 1: Phylogenetic tree calculated on the basis of ORF2 sequences of porcine circovirus 2 (PCV-2) using the Neighbor Joining algorithm of the PHYLIP package [25] with 1000 bootstrap replicates. Bootstrap values over 500 are shown as $1 / 10$ of the value. Argentinean isolates are identified in red. Reference strains are indicated with the corresponding GenBank accession number and the country and year of report. Isolates are grouped by subgenotypes as described by Fort et al. 2007 [23] and separated by subgroups by the dashed line as described by Wiederkehr et al. 2008 [28]. 
PCV-2 genotype. Only samples from 2003 to 2008 with confirmatory diagnosis of PCVAD were processed. Sequence and phylogenetic analyses clustered the PCV-2b group with a common A-B subgroup, with an identity of 99.8\% [15]. The reason we named a new cluster A-B is because bootstrap values were not suitable enough, based on the sequences used in this study, to support the differentiation between clusters $\mathrm{A}$ and $\mathrm{B}$. The PCV-2b sequences identified were very similar to an isolate previously reported in Argentina in 2005 (Genbank EF458306). The Argentinean PCV-2b genotype isolates were found to be closely related to some isolates from Brazil 2004, North America 2005, Europe 20032004, and China 2002. In addition, all sequences present the 1486 motif TcA/aac/CCC/CGC belonging to the PCV-2b genotype described by Cheung et al. 2007 [20], and contain amino acid substitutions similar to those described by Grau-Roma et al. 2008 [16]. In Canada, PCV-2b isolates from PMWS outbreaks in 2005 share an interval identity between 99\% and 100\% [19]. However, other studies have identified distinctive genotypes from PCVAD samples [28, 32] and a variable identity within each genotype [22].

In Argentina, the first outbreak of PMWS was reported in 2002 [11] and thereafter clinical PCVAD emerged as emerging diseases. Our study comprised PCVAD samples taken from 2003 to 2008 and the results suggest that PCV$2 \mathrm{~b}$ is the predominant genotype in Argentina and that it could be associated with a systemic form of PCVAD, namely, PMWS. In Argentina, serological or immunohistochemical retrospective studies on PCV-2 have not been carried out and, therefore it remains unknown how and when PCV$2 \mathrm{~b}$ was introduced in the Argentinean herds. Other sources of infection such as inappropriate used of vaccines can be ruledout because only inactivated PCV-2 and PCV-2 ORF2 protein vaccines have been licensed.

In summary, the present study contributes to the knowledge on the distribution of PCV-2 genotypes circulating in Argentina. Our findings may also help to establish a base of information to study the emergence of new viral variants in this region.

\section{Acknowledgments}

The authors would like to thank Andrea Puebla and her group for their technical sequencing support. This paper was partially supported by Proyecto Específico INTA Enfermedades de los Porcinos (AESA203921), by the European Community (Proyecto Integrado Cadena Carne Aviar-BiotecSur), by the Ministerio de Ciencia, Tecnologia e Innovacion Productiva from Argentina (BID-PICT 2005-33987), and by Secretaría de Ciencia y Técnica, Universidad Nacional de La Plata.

\section{References}

[1] I. Tischer, H. Gelderblom, W. Vettermann, and M. A. Koch, "A very small porcine virus with circular single-stranded DNA," Nature, vol. 295, no. 5844, pp. 64-66, 1982.

[2] "The circoviridae," in Virus Taxonomy Sixth Report of the International Committee on Taxonomy of Viruses, P. Lukert, G.
F. De Boer, J. L. Dale et al., Eds., pp. 166-168, Springer, Vienna, Austria, 1995.

[3] B. M. Meehan, J. L. Creelan, M. S. McNulty, and D. Todd, "Sequence of porcine circovirus DNA: affinities with plant circovirses," Journal of General Virology, vol. 78, no. 1, pp. 221227, 1997.

[4] I. Tischer, R. Rasch, and G. Tochtermann, "Characterization of papovavirus and picornavirus like particles in permanent pig kidney cell lines," Zentralblatt für Bakteriologie, vol. 226, no. 2, pp. 153-167, 1974.

[5] J. C. Harding, "Post-weaning multisystemic wasting syndrome (PMWS): preliminary epidemiology and clinical presentation," in Proceedings of the 27th Annual Meeting Western Canadian Association of Swine Practitioners, p. 21, Saskatoon, Canada, October 1996.

[6] J. Ellis, S. Krakowka, M. Lairmore et al., "Reproduction of lesions of postweaning multisystemic wasting syndrome in gnotobiotic piglets," Journal of Veterinary Diagnostic Investigation, vol. 11, no. 1, pp. 3-14, 1999.

[7] J. Segales, M. Domingo, and K. S. Latimer, "Porcine circovirus is present in cases of porcine dermatitis and nephropathy syndrome (PDNS)," in Proceedings of the 15th International Pig Veterinary Society Congress, p. 215, Birmingham, UK, 1998.

[8] J. Segales, C. Rosell, and M. Domingo, "Pathological findings associated with naturally acquired porcine circovirus type 2 associated disease," Veterinary Microbiology, vol. 98, no. 2, pp. 137-149, 2004.

[9] R. Cariolet, B. Blanchard, M. Le Dimna et al., "Consequences of PCV-2 experimental infection of non immune SPF sows using the intrauterine route," in Proceedings of the International Conference on ssDNA Viruses of Plants, Birds and Primates, p. 129, Saint-Malo, France, 2001.

[10] T. Opriessnig, X. J. Meng, and P. G. Halbur, "Porcine circovirus type 2-associated disease: update on current terminology, clinical manifestations, pathogenesis, diagnosis, and intervention strategies," Journal of Veterinary Diagnostic Investigation, vol. 19, no. 6, pp. 591-615, 2007.

[11] J. Sarradell, A. M. Perez, M. Andrada, F. Rodriguez, A. Fernandez, and J. Segales, "PMWS in Argentina," Veterinary Record, vol. 150, no. 10, p. 323, 2002.

[12] J. C. Harding and E. G. Clark, "Recognizing and diagnosing postweaning multisystemic wasting syndrome (PMWS)," Journal of Swine Health and Production, vol. 5, no. 5, pp. 201203, 1997.

[13] C. Rosell, J. Segales, J. Plana-Duran et al., "Pathological, immunohistochemical, and in-situ hybridization studies of natural cases of postweaning multisystemic wasting syndrome (PMWS) in pigs," Journal of Comparative Pathology, vol. 120, no. 1, pp. 59-78, 1999.

[14] A. Mankertz, R. Caliskan, K. Hattermann et al., "Molecular biology of Porcine circovirus: analyses of gene expression and viral replication," Veterinary Microbiology, vol. 98, no. 2, pp. 81-88, 2004.

[15] A. Olvera, M. Cortey, and J. Segales, "Molecular evolution of porcine circovirus type 2 genomes: phylogeny and clonality," Virology, vol. 357, no. 2, pp. 175-185, 2007.

[16] L. Grau-Roma, E. Crisci, M. Sibila et al., "A proposal on porcine circovirus type 2 (PCV2) genotype definition and their relation with postweaning multisystemic wasting syndrome (PMWS) occurrence," Veterinary Microbiology, vol. 128, no. 1-2, pp. 23-35, 2008.

[17] J. Segalés, A. Olvera, L. Grau-Roma et al., "PCV-2 genotype definition and nomenclature," Veterinary Record, vol. 162, no. 26, pp. 867-868, 2008. 
[18] W. Cui, G. Xin, X. Ge, and H. Yang, "Genotype analysis of Chinese porcine circovirus type 2 in 2008-2009," in Proceedings of the 21st International Pigs Veterinary Society Congress, p. 322, Vancouver, Canada, 2010.

[19] C. A. Gagnon, D. Tremblay, P. Tijssen, M. H. Venne, A. Houde, and S. M. Elahi, "The emergence of porcine circovirus $2 \mathrm{~b}$ genotype (PCV-2b) in swine in Canada," Canadian Veterinary Journal, vol. 48, no. 8, pp. 811-819, 2007.

[20] A. K. Cheung, K. M. Lager, O. I. Kohutyuk et al., "Detection of two porcine circovirus type 2 genotypic groups in United States swine herds," Archives of Virology, vol. 152, no. 5, pp. 1035-1044, 2007.

[21] M. De Castro, A. Cortez, M. B. Heinemann, P. E. Brandao, and L. J. Richtzenhain, "Genetic diversity of Brazilian strains of porcine circovirus type $2(\mathrm{PCV}-2)$ revealed by analysis of the cap gene (ORF-2)," Archives of Virology, vol. 152, no. 8, pp. 1435-1445, 2007.

[22] J. R. Ciacci-Zanella, N. L. Simon, L. S. Pinto et al., "Detection of porcine Circovirus type 2 (PCV2) variants PCV2-1 and PCV2-2 in Brazilian pig population," Research in Veterinary Science, vol. 87, no. 1, pp. 157-160, 2009.

[23] M. Fort, A. Olvera, M. Sibila, J. Segales, and E. Mateu, "Detection of neutralizing antibodies in postweaning multisystemic wasting syndrome (PMWS)-affected and non-PMWS-affected pigs," Veterinary Microbiology, vol. 125, no. 3-4, pp. 244-255, 2007.

[24] J. D. Thompson, D. G. Higgins, and T. J. Gibson, "CLUSTAL $\mathrm{W}$ : improving the sensitivity of progressive nultiple sequence alignment through sequence weighting, position-specific gap penalties and weight matrix choice," Nucleic Acids Research, vol. 22, no. 22, pp. 4673-4680, 1994.

[25] J. Felsenstein, "PHYLIP* phylogeny interference package (version 3.2)," Cladistics, vol. 5, pp. 164-166, 1989.

[26] D. M. Hillis and J. J. Bull, "An empirical test of bootstrapping as a method for assessing confidence in phylogenetic analysis," Systematic Biology, vol. 42, no. 2, pp. 182-192, 1993.

[27] R. D. page, "Treeview: an application to display phylogenetic trees on personal computers," Computer Applications in the Biosciences, vol. 12, no. 4, pp. 357-358, 1996.

[28] D. D. Wiederkehr, T. Sydler, E. Brugnera, A. Pospischil, E. Buergi, and X. Sidler, "Pathogenic differences of porcine circovirus type 2 genotypes in Switzerland," in Proceedings of the 20th International Pigs Veterinary Society Congress, p. 26, Durban, South Africa, 2008.

[29] J. C. Harding, J. A. Ellis, K. A. McIntosh, and S. Krakowka, "Dual heterologous porcine circovirus genogroup 2a/2b infection induces severe disease in germ-free pigs," Veterinary Microbiology, vol. 145, no. 3-4, pp. 209-219, 2010.

[30] K. M. Lager, P. C. Gauger, A. L. Vincent, T. Opriessnig, M. E. Kehrli Jr., and A. K. Cheung, "Mortality in pigs given porcine circovirus type 2 subgroup 1 and 2 viruses derived from DNA clones," Veterinary Record, vol. 161, no. 12, pp. 428-429, 2007.

[31] T. Opriessnig, J. R. Prickett, D. M. Madson et al., "Porcine circovirus type 2 (PCV2)-infection and re-inoculation with homologous or heterologous strains: virological, serological, pathological and clinical effects in growing pigs," Veterinary Research, vol. 41, no. 3, p. 31, 2010.

[32] J. S. Chae and K. S. Choi, "Genetic diversity of porcine circovirus type 2 from pigs in Republic of Korea," Research in Veterinary Science, vol. 88, no. 2, pp. 333-338, 2010. 

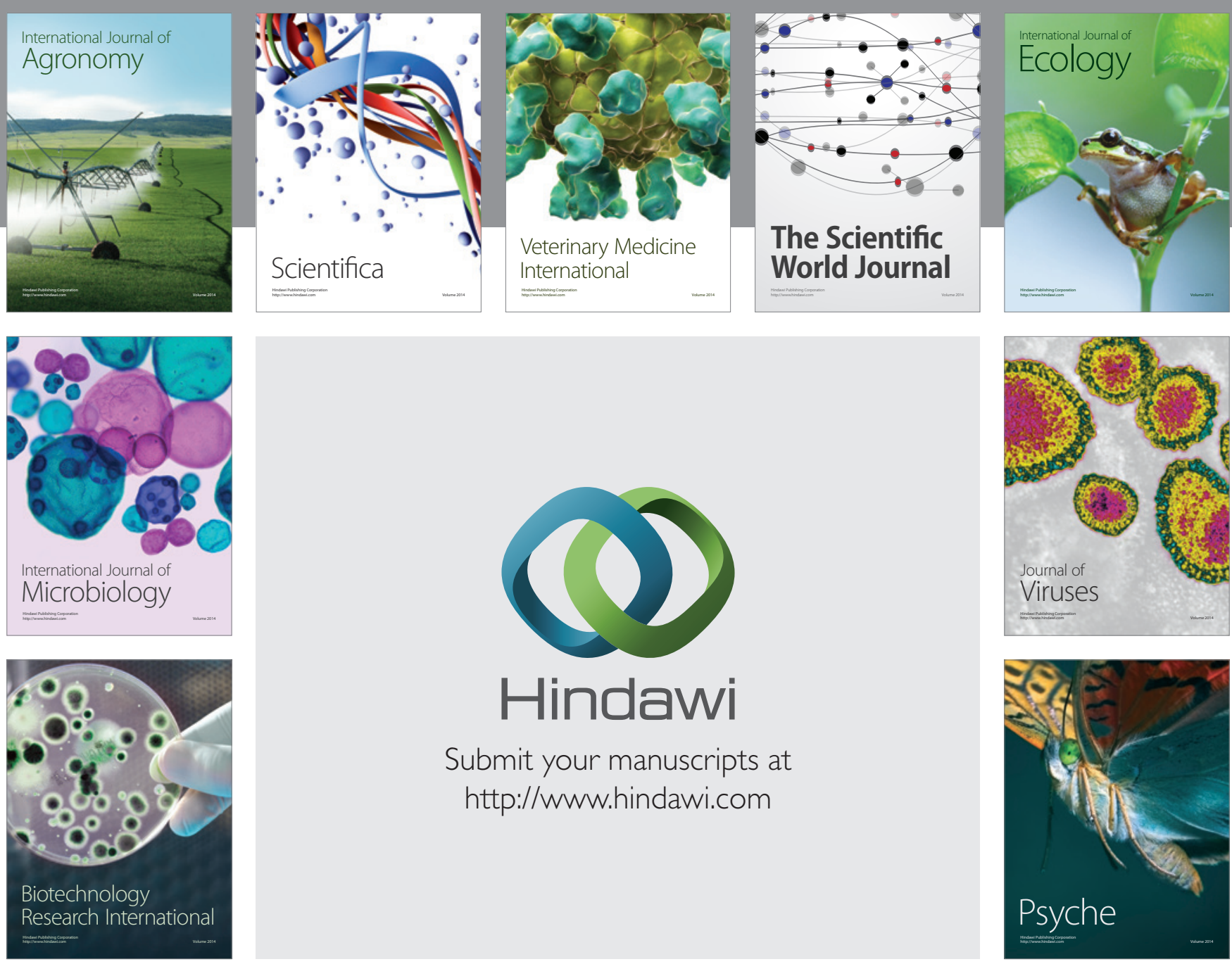

Submit your manuscripts at http://www.hindawi.com
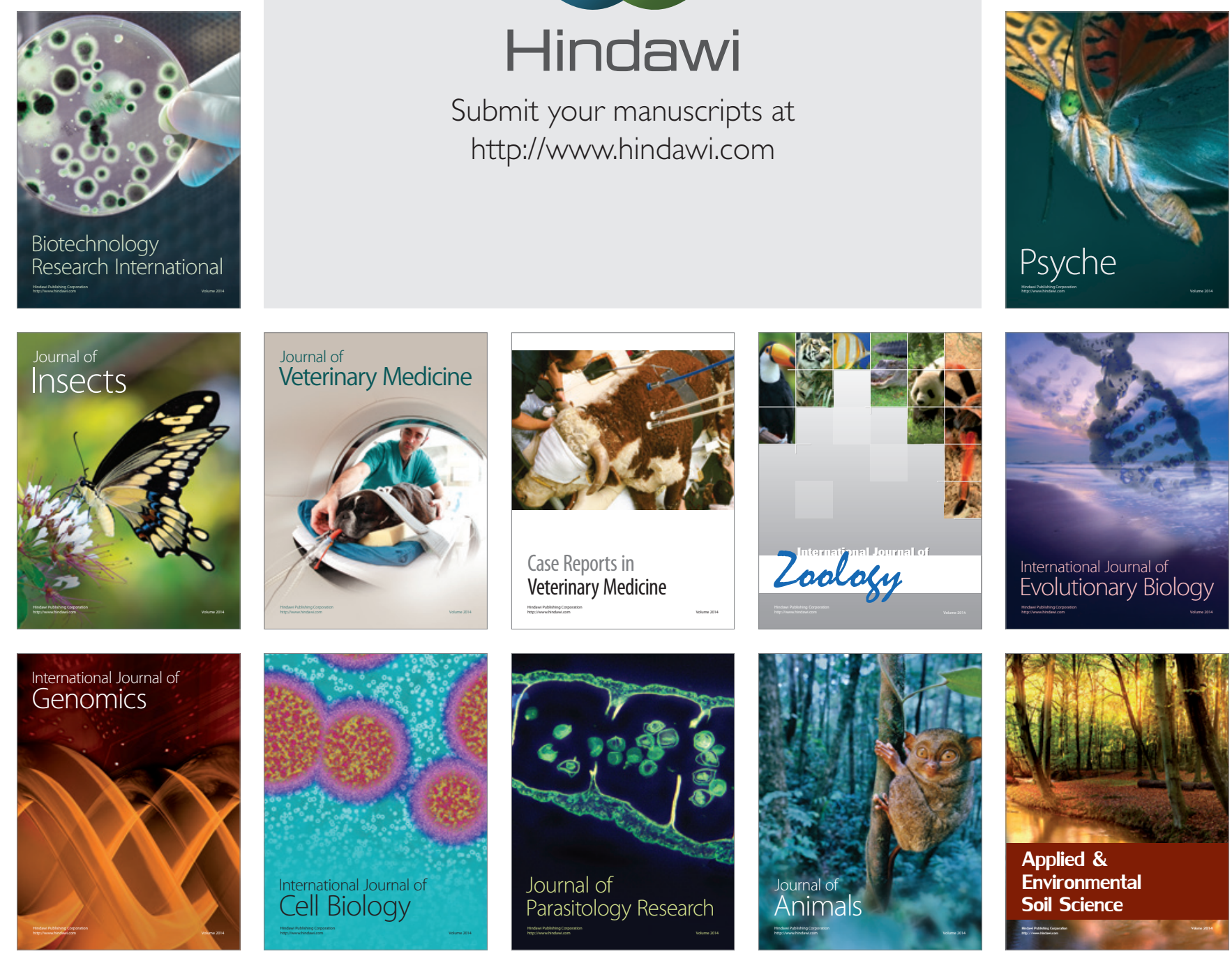